\title{
Les enjeux de la légalité dans "Phèdre"
}

\section{Ralph Albanese}

\section{(2) OpenEdition}

\section{Journals}

\section{Édition électronique}

URL : http://journals.openedition.org/studifrancesi/14587

DOI : 10.4000/studifrancesi. 14587

ISSN : 2427-5856

\section{Éditeur}

Rosenberg \& Sellier

\section{Édition imprimée}

Date de publication : 1 décembre 2018

Pagination : 416-424

ISSN : 0039-2944

\section{Référence électronique}

Ralph Albanese, "Les enjeux de la légalité dans "Phèdre" », Studi Francesi [En ligne], 186 (LXII | III) | 2018, mis en ligne le 01 décembre 2019, consulté le 06 janvier 2021. URL : http://

journals.openedition.org/studifrancesi/14587 ; DOI : https://doi.org/10.4000/studifrancesi.14587

\section{(c) (i) (9)}

Studi Francesi è distribuita con Licenza Creative Commons Attribuzione - Non commerciale - Non opere derivate 4.0 Internazionale. 


\title{
Les enjeux de la légalité dans "Phèdre"
}

\begin{abstract}
Phedre illustrates the problematic of the Law and its repression. The protagonists find themselves in a universe governed by moral anarchy and are therefore prompted to subvert the Law. Since they define themselves in terms of their transgressive behavior, Hippolyte, Phèdre and Thésée struggle with the radical impossibility of controlling their desire. To the extent that the legal system weighs on their conscience, they are vulnerable to intense feelings of guilt. Given that Phèdre highlights various paradigms of the Law (= purity) and its violation (= impurity), the protagonists must confront an irremediable conflict between their libidinal desire (= concupiscence) and their conscience (= superego). Through the intermediary of social taboos designed to repress sexual passion, the emergence of the Law results in the subjection of the individual, and it is useful to shed light on the mediation of sexuality within the confines of the Law: the patriarchal order seeks above all to repress sexual excess. A multiplicity of laws have a negative impact on the protagonists (legislation against suicide and incest, on marital fidelity and duties towards the well-being of children). Such an undifferentiated mass of laws obsesses, in varying degrees, Hippolyte, Phèdre and Thésée, who are confronted with the central question of whether or not these laws should be violated. This article examines both the complementary and the mutually-exclusive relationships between Law and Desire in Phèdre. It also demonstrates the broad lexical implications of the Law and criminality in Racine's final secular tragedy.
\end{abstract}

À la suite de son évasion du royaume des morts, Thésée, surpris par le tyran de l'Épire, «sans défense et sans arme» (III, 5, v. 961'1), s'aperçoit du dilemme dans lequel il se trouve. À l'instar du roi d'Athènes, Hippolyte et Phèdre se situent dans un univers moralement anarchique, d'où leur volonté commune de subvertir la Loi. Se définissant par rapport à leurs démarches transgressives, les protagonistes se heurtent à l'impossibilité radicale de dompter leurs désirs. La légalité pesant sur leur conscience, ils s'avèrent en proie à une culpabilité intense. Dans la mesure où, comme nous allons le voir, Phèdre met en évidence les paradigmes de la Loi (pureté) et du dérèglement (impureté), on assiste, chez eux, à un conflit irrémédiable entre les pulsions libidinales (la concupiscence) et la conscience morale (le sur-moi). L'émergence de la Loi, par l'intermédiaire des tabous sociaux visant à refréner l'amour-passion, donne lieu à l'assujettissement du personnage, et M. Greenberg s'interroge à juste titre sur la médiation de la sexualité au sein de la Loi²: l'Ordre patriarcal doit réprimer la démesure sexuelle. On a affaire ici à une multiplicité de lois qui exercent une influence néfaste sur les protagonistes: lois contre le suicide et contre l'inceste, loi sur la fidélité conju-

(1) Toutes nos citations de la pièce renvoient à l'édition de G. Forestier, Racine. Euvres complètes, vol. 1, Paris, Gallimard, «Pléiade», 1999.

(2) M. GReEnBerg, Subjectivity and Subjugation in Seventeenth-Century Drama and Prose, Cambridge, Cambridge University Press, 1992, p. 168. 
gale et les devoirs envers les enfants ${ }^{3}$. On assiste, en fait, à une masse indifférenciée des lois: enfreindre ou ne pas enfreindre les lois, telle est la question qui hante, à des degrés divers, Phèdre, Hippolyte et Thésée. Nous voudrions examiner les rapports de complémentarité et d'exclusion entre la Loi et le Désir qui se manifestent dans Phèdre $e^{4}$. Plus précisément, nous espérons mettre en évidence l'ampleur lexicale de la Loi et de la criminalité - le discours tragique du licite et de l'illicite - dans la dernière tragédie profane de Racines.

«Implacable ennemi des amoureuses lois» (v. 59), le «superbe» Hippolyte s'en prend avec vigueur aux lois impérieuses de Vénus. Jusqu'ici, il s'est mis en guerre contre le «joug» amoureux (v. 60) alors que son père n'a que trop bien obéi aux impératifs de la déesse de l'Amour. Se présentant en quelque sorte en psychologue, Théramène décèle un changement significatif dans la personnalité d'Hippolyte. S'interrogeant sur sa volonté de fuite, il considère la résistance tenace d'Hippolyte aux lois de l'Amour comme un péché suprême. Il laisse entendre, en plus, que ce fils ne doit plus condamner l'inconstance de son père et justifie, par là, paradoxalement, les entreprises amoureuses de Thésée ${ }^{6}$ (vv. 61-62). En reprochant à Hippolyte de vouloir se situer au-delà des limites de la condition humaine, son précepteur signale que l'on ne peut guère mépriser Vénus avec impunité. La réaction défensive d'Hippolyte marque une répugnance à tout effort pour l'apprivoiser (vv. 65-68). Sa fierté le place, de toute évidence, au-dessus de l'humanité commune. Faisant figure d'ennemi exemplaire des femmes, ce fils d'une Amazone s'avère marqué par une insensibilité génétique. Face à Théramène, il s'évertue à nier son amour illégitime en réprimant son désir sexuel, d'où son «dessein» (v. 1), c'est-à-dire, son vœu constant de fuite.

Instrument de la Loi patriarcale, Thésée a purgé le monde des brigands et des monstres afin de fonder la civilisation. D'autre part, désireux de mettre fin à la dynastie des Pallantides, Vénus fait le vœu d'éteindre la race de Pasiphaé et de ses descendants. Divinité malveillante, elle s'acharne à poursuivre toutes les filles du Soleil, c'est-à-dire, la famille de Minos: Pasiphaé, Ariane et Phèdre. Relevant d'une lignée maudite, «la fille de Minos et de Pasiphaé» est la proie de ses pulsions dénaturées. On a affaire à un déterminisme héréditaire, autant dire la loi de l'atavisme, car elle porte un instinct dépravé. Tel Néron ${ }^{7}$, elle souffre du poids de son hérédité criminelle: «Que ces vains ornements, que ces voiles me pèsent!» (v. 158). S'avérant l'objet d'une malédiction divine, Phèdre est mue par un désir transgressif, hors norme, qui fait violence aux «amoureuses lois». Écrasée par l'ampleur de sa passion illégitime, elle se montre tiraillée entre la culpabilité, la honte et le désir. Cette héroïne, la première chez Racine à s'apercevoir des conséquences néfastes de sa passion, n’ignore point ses démarches criminelles. Soumise à des impératifs contradictoires (le Soleil/ Vénus), elle témoigne d'une filiation profonde entre la Loi et le Désirr. Sa faute est

(3) E. Zimmerman, La Liberté et le destin dans le théâtre de Jean Racine, Saratoga, CA, Anma Libri, 1982, p. 90 .

(4) Voir, à cet égard, F. Orlando, Lecture freudienne de "Phèdre", Paris, Les Belles Lettres, 1986, p. 100.

(5) Il convient de se reporter à l'article éclairant de L. Horowitz, Racine's Laws, «Dalhousie French Studies» 49, 1999, p. 132.

(6) D'après le "discours naturaliste" de Théramène, tout mortel doit reconnaître la souveraineté de la loi de l'amour. X. Darcos, Racine: "Phèdre", Paris, Hachette, 1991, p. 4.

(7) Agrippine met en valeur ainsi l'hérédité inquiétante de son fils: «... je lis sur son visage/Des fiers Domitius l'humeur triste et sauvage» (Britannicus, I, 1, vv. 35-36).

(8) J. Rohou met en évidence le décalage entre la conscience et la pulsion dans Phèdre: «Vénus est la pulsion, le Soleil la conscience, le monstre final est l'image du vœu monstrueux de Thésée et surtout de la concupiscence monstrueuse de Phèdre - dont cet "indomptable taureau" rappelle le demi-frère, le Minotaure - voire de la concupiscence qui s'est emparée d'Hippolyte et l'empêche de maîtriser désormais ses chevaux, autrement dit ses pulsions» (J. Racine, Bilan critique, Paris, Nathan, 1994, pp. 96-97). 
doublement condamnable à cause de son lignage paternel, qui devait le placer dans une droiture morale rigoureuse, voire hypertrophiée:

... «fille de Minos et de Pasiphaé» (1. 1. 36), Phèdre tient aussi d'un père droit et vertueux juge aux enfers - «mon père y tient l'urne fatale» (4. 6. 1278) - et incarnation des valeurs civilisées. À une sexualité débridée Phèdre joint donc la légalité; elle réunit en elle-même la licence et la culpabilité, l'instinct et la répression'.

En remontant à la source de son mal, Phèdre évoque les conditions dans lesquelles elle s'est engagée dans un mariage soumis à la règle patriarcale en s'assujettissant aux «lois de l'hymen» (v. $\left.270^{10}\right)$. Il convient de noter que le contrat de mariage suppose en principe que Thésée et Phèdre fassent partie d'une alliance légale aux obligations mutuelles. S'il est vrai que cet engagement formel s'avère compromis dès que Phèdre voit son «superbe ennemi» (v. 272), tout se passe comme si elle se croyait heureuse et sauve grâce à son mariage. Par un effort délibéré de sa volonté (cf. «Par des vœux assidus...» v. 279), elle s'est évertuée à détourner «ses feux redoutables» et «(ses) tourments inévitables» (vv. 277-278). Désireuse de conserver sa vertu d'épouse, elle s'applique à exiler Hippolyte et à bannir sa réalité en défendant - grâce à «une expresse loi» (vv. 603-604), autant dire une ordonnance discursive - l'évocation de son nom ${ }^{11}$. Vouloir codifier une loi qui défend la nomination de son objet libidinal, c'est dévoiler la faiblesse intrinsèque de la Loi face au Désir. Aussi Phèdre se livre-telle à une contre-offensive en finissant par se sentir innocente en raison de l'absence d'Hippolyte. Phèdre parvient donc à se créer une distance psychologique en évitant l'objet de son désir et se maîtrise en feignant d'être injuste envers lui: de persécutée, elle devient persécutrice (vv. 293-294). Se jouant ainsi d'elle-même, elle fait preuve de mauvaise foi. Plus précisément, la Loi lui servant à contrôler ses comportements déréglés, elle réussit à prendre des mesures décisives pour anéantir sa passion coupable. Elle se soucie d'ailleurs de compenser la vie débauchée de sa mère en menant une existence sans reproche. De toute évidence, l'amour a fait irruption dans le mariage récent de Phèdre et cette passion finit par subvertir «les lois de l'hymen». Ironiquement, c'est Thésée, par imprudence, qui a lui-même ramené sa femme en présence d'Hippolyte - son «superbe ennemi» qu'elle avait voulu éloigner (v. 272) - à Trézène (vv. 929-931).

À la nouvelle de la mort de son père, Hippolyte se livre à un choix impropre en «oubliant» la loi politique de Thésée (v. 327). En convoitant la captive de son père, son amour pour Aricie s'apparente à un crime d'État. D'après l'interdit politique de Thésée, Hippolyte se devait d'ignorer Aricie. Opérant une révocation des lois patriarcales ayant pour but la libération d'Aricie, il lui enlève la tutelle rigoureuse imposée par Thésée, c'est-à-dire, la sévérité des lois de chasteté auxquelles elle était soumise. S'apercevant de l'impureté de son sang, Hippolyte s'en prend à cette «superbe loi» qui lui défend d'occuper le trône d'Attique (v. 492). Désireux d'apparaître «plus légitime», il estime que ses «droits» l'emportent sur une légalité inconséquente (cf. le «caprice des lois», v. 492). Par ailleurs, il se prévaut de ses droits auprès de

(9) J. BRody, Freud, Racine et la connaissance tragique, dans «Lectures classiques», Charlottesville, Rookwood Press, 1996, p. 230.

(10) Conformément aux valeurs inhérentes à l'Ordre patriarcal, les lois conjugales «témoignent de la répression des femmes», c'est-à-dire, la soumission de la femme à son mari (M.-F. BRUNEAU, Racine, le jansénisme et la problématique de la modernité, Paris, Corti, 1986, p. 344).

(11) Se reporter à B. HöFER, Psychosomatic Disorders in Seventeenth-Century French Literature, Surrey, Ashgate, 2009, p. 197. 
son demi-frère, le fils de Phèdre, et l'on songe au rapport fratricide entre Néron et Britannicus $^{12}$. Ainsi, en révoquant la loi dynastique portant sur la succession au trône, Hippolyte témoigne d'un mépris souverain du légalisme attique, d'où le contraste entre les droits humains et l'injustice des lois (vv. 491-492).

Ayant hérité le désir déréglé de sa mère, Phèdre finit par dépasser les bornes légitimes, c'est-à-dire, les limites raisonnables du Désir. Elle s'avère mue par une force irrationnelle et démesurée. Par suite de l'envoûtement de sa passion, elle est la «proie» de Venus: «C'est Vénus tout entière à sa proie attachée» (v. 306); «(sa) flamme si noire» se ramène donc à sa passion dénaturée (v. 310). Il va de soi qu'une hérédité funeste livre l'héroïne tragique aux affres de sa libido sentiendi et elle s'efforce de réprimer son amour adultère. On assiste chez elle à l'antinomie fondamentale entre le désir et la répression. Une telle répression de la faute est liée d'ailleurs à l'effet étouffant de son secret: «Phèdre, atteinte d'un mal qu'elle s'obstine à taire» (v. 45). Le refoulement de son désir provoque aussi des désordres corporels (cf. vv. 153-157). Remarquons en plus que son aveu à Enone constitue non seulement son premier geste transgressif mais aussi une erreur fatidique (I, 3). Dans son égarement passionnel, Phèdre se montre incapable de nommer Hippolyte et doit recourir au style détourné de la périphrase («ce fils de l'Amazone», v. 262) qui aboutit au «C'est toi qui l'as nommé» (v. 264), projetant ainsi la faute sur CEnone. De plus, elle finit par ériger son «ennemi» en un dieu (v. 293). Conformément à Néron qui idolâtrait Junie (Britannicus, II, 2, v. 384), elle adore Hippolyte avec une passion idolâtre. S'étant déchargée alors de son péché, Phèdre se transforme en objet d'une réprobation morale: «J'ai pris la vie en haine et ma flamme en horreur» (v. 308).

Avant de passer à l'aveu que fait Phèdre à Hippolyte (II, 5), il faut tenir compte de la valeur dramaturgique de la première péripétie de la pièce, à savoir, la (fausse) nouvelle de la mort de Thésée ${ }^{13}$. L'action tragique va se dérouler en fonction de cette communication erronée, et l'on assiste à une volonté commune, du moins de la part de Phèdre et Enone, de croire à la véracité de la nouvelle de Panope: il s'agit, en fait, d'une illusion que l'on cultive. Le problème dynastique de Phèdre se pose alors puisque plusieurs trônes ne sont pas occupés, et Trézène, Crète et Athènes se trouvent dépourvus d'autorité légale. Au demeurant, la mort du Roi laisse croire au relâchement des lois civiles et canoniques. Dans la mesure où Thésée incarne la justice royale, son absence aux deux premiers actes de Phèdre suppose non seulement la précarité des normes culturelles mais aussi la faillite de toute autorité légale et morale $^{14}$. La disparition du Père crée ainsi une inquiétude réelle dans le royaume et aboutit au dépérissement sinon à l'effacement de sa Loi. Chose plus importante, peut-être, l'absence du Père entraîne le déchaînement des passions.

Son mari porté disparu, Phèdre se considère alors veuve. Tout se passe comme si la «mort» de Thésée rendait son amour innocent. Son nouveau statut de veuve finit, en effet, par la disculper (I, 5). Bien qu'elle se croie sincèrement veuve, sa perception s'avère, de toute évidence, mal fondée. Phèdre se nourrit donc de l'illusion de la liberté. Son deuxième aveu criminel, cette fois-ci à Hippolyte, se révèle tout aussi irréversible que celui qu'elle a adressé à CEnone: «... tu m’as trop entendu» (v. 670); «... je n'ai que trop parlé» (v. 740) (nous soulignons), d'où l'impossibilité de faire un retour en arrière. S'adressant à l'imaginaire d'Hippolyte, la reine crétoise l'engage

(12) Outre le récit de l'empoisonnement de Britannicus (V, 5), on peut citer l'aveu cruel de l'empereur: «J'embrasse mon rival pour mieux l'étouffer» (IV, 3, v. 1314).

(13) La formule qu'adresse Panope à Phèdre est fort paradoxale: «La mort vous a ravi votre invincible époux», v. 319 (nous soulignons).

(14) L. Horowitz, Racine's Laws cit., p. 132. 
dans une action interdite: elle témoigne de la transformation fantasmatique de Thésée en Hippolyte. Alors que ce dernier inscrit la douleur de Phèdre dans l'ordre commun (il est normal que les marâtres soient jalouses...) (vv. 612-613), Phèdre cherche à se distinguer de «cette loi commune» (v. 616). Ensuite, il faut noter que l'aphorisme de Phèdre est fondé sur une erreur logistique: «On ne voit point deux fois le rivage des morts» (v. 623), car il prend la mort de Thésée pour une certitude. Dans l'égarement passionnel de la reine, son fantasme («Toujours devant mes yeux je crois voir mon époux») suppose une vision de la réalité inauthentique (vv. 628-630). À la quête d'un Thésée idéalisé, elle évoque le caractère libidinal d'un jeune Thésée: «... fidèle... fier, et même un peu farouche,/Charmant, jeune, traînant tous les cœurs après soi» (vv. 638-639), puis elle met en accusation les dieux (vv. 679-680). Humiliée et troublée, constatant l'indifférence d'Hippolyte dans ses yeux, elle ne peut s'empêcher de chercher encore son regard («Si tes yeux un moment pouvaient me regarder», v. 692), évoquant par là l'avanie d'Hermione face à l'apathie de Pyrrhus (Andromaque, IV, 5). S'assimilant à un monstre (v. 701), Phèdre se nomme périphrastiquement «La veuve de Thésée»: veuve, elle serait libre d'aimer Hippolyte. Toutefois, elle s'avère subjectivement coupable d'inceste (v. 702).

Au tabou d'inceste s'ajoute, chez l'héroïne, le crime politique de ne pas reconnaître le roi légitime. En fait, elle ne saurait maîtriser le corps politique de l'État ${ }^{15}$. Dans la mesure où elle s'est détournée de la Loi, elle ne saurait régner sur le plan politique puisque la maîtrise de soi lui fait défaut: «Moi régner! Moi, ranger un État sous ma loi» (v. 759). Le moi anarchique de la reine s'avérant incapable de régner, elle est plutôt mue par la loi de l'instinct (= le «ça,» ou bien la pulsion libidinale). Phèdre reste à tel point enfermée dans son «erreur passionnelle» ${ }^{16}$ qu'elle finit, selon Enone, par pervertir sa fonction maternelle en raison de son rapport avec Hippolyte: «Vous trahissez enfin vos enfants malheureux, | Que vous précipitez sous un joug rigoureux» (vv. 199-200). À ce propos, il convient de citer C. Venesoen, qui fait le bilan des mères dénaturées chez Racine (y compris Agrippine, Phèdre et Athalie) ${ }^{17}$. Comme on le verra, Phèdre touche au comble du sentiment de la faute à l'Acte IV. Craignant le jugement infernal de Minos, elle voudrait se discupler devant lui (vv. 1289-1290). Ainsi, C. Mauron discerne, chez elle, la mise en place d'une scène de comparution devant un juge:

Chaque acte comporte... une 'comparution' de Phèdre: I - devant CEnone; II - devant Hippolyte; III - devant Thésée; IV - devant Minos; V - devant Thésée. En dépit des variations évidentes, on peut toutes les superposer, car toutes sont... ressenties, vécues par Phèdre comme des comparutions devant un juge ${ }^{18}$. Le psychocritique évoque aussi le phénomène de «disculpation» chez Phèdre, c'est-à-dire, la faute étant rejetée sur «Le ciel» (v. 1625) ou bien sur «La détestable Enone» (v. 1626).

Au nom de la morale, CEnone accuse sa maîtresse de transgresser la loi religieuse ainsi que son devoir conjugal et maternel (I, 3). Dès l'annonce de la mort de Thésée (I, 4), grâce aux conseils de sa nourrice, Phèdre s'engage dans une voie nettement interdite. Le pragmatisme d'Enone offre à la reine une alternative réelle. Mue par les valeurs anti-tragiques, elle s'enferme dans une illusion et s'applique à transformer

(15) Selon L. Horowitz, la reine met en question ainsi la légitimité de la loi dynastique (ibid., p. 137).

(16) Voir à ce sujet C. Puzin, "Phèdre". Jean Racine, Paris, Nathan, 1990, p. 47.

(17) Aspects de la relation filiale dans "Phèdre", sous la direction de R.L. BARNETT, éd. Re-Lectures raciniennes, Paris, Biblio 17, 1986, p. 224.

(18) Ch. Mauron, Phèdre, Paris, Corti, 1968, p. 105. 
la faute tragique en une erreur anodine (cf. «une excusable erreur», v. 1296). On ne saurait trop insister sur la complicité d'CEnone dans le désir transgressif de Phèdre. Plus précisément, la nourrice suggère à sa maîtresse que la mort de Thésée entraîne une nouvelle légalité (cf. «d'autres lois», v. 340), et fait ressortir la légitimité de son amour (cf. «une flamme ordinaire», v. 350). On assiste alors à une rupture définitive des liens du mariage (= des vœux de fidélité) qui finit par situer la reine au-delà des «lois de l'hymen». Par-là, elle cultive, chez sa maîtresse, un espoir mal fondé. D'après elle, la mort du Roi a «dénoué» en quelque sorte le drame de sa passion illicite: «Thésée en expirant vient de rompre les nœuds» (v. 351). De «scandaleuse», la flamme de Phèdre s'inscrit alors dans les normes de l'humanité commune. Cette flamme se trouve, dès lors, non seulement banalisée mais aussi déculpabilisée. Notons, enfin, qu'Enone fait appel maintenant aux sentiments maternels de Phèdre (vv. 343-348). Il faut souligner, toutefois, la responsabilité morale de l'héroïne qui, malgré son état affaibli, autorise sa nourrice à agir.

Avant d'aborder la démesure criminelle d'CEnone vis-à-vis de Thésée (IV, 1), force est de mettre en évidence la valeur dramaturgique de la deuxième péripétie de Phèdre, à savoir, le retour du Roi. En tant que législateur suprême du royaume, Thésée détient l'autorité politique, paternelle et conjugale. À cela s'ajoute évidemment son rôle justicier. Symbole de l'Ordre patriarcal, la réapparition de Thésée suppose, en effet, la réaffirmation de la Loi. De même que le bruit de sa mort, son retour imprévu relève de l'univers de la contingence. Ce retournement de situation sert à déclencher le mécanisme tragique qui aboutit à la catastrophe. Outre le suicide de Phèdre, le retour inopiné du Père entraîne son fils dévoué dans l'univers de la transgression à cause de son amour interdit avec Aricie, et fait sentir à Hippolyte le sentiment de la faute en aiguisant sa culpabilité. Il va de soi que la calomnie d'EEnone plonge Thésée dans un état d'égarement: «Je ne sais où je vais, je ne sais où je suis» (IV, 1, v. 1004). Se persuadant facilement du dessein maléfique de son fils, le Roi déchiffre mal le silence de sa femme (v. 1013). Tout porte à croire, d'abord, que les tendances libidinales de Thésée le prédisposent à donner créance à la délation d'CEnone. De toute évidence, le Roi fait preuve d'une erreur de jugement (bamartia), et l'on s'étonne de la promptitude avec laquelle il prête croyance au mensonge d'une servante. Père offensé, il juge hâtivement, et son jugement précipité met en évidence la capacité du Père justicier à être trompé. Bref, sa faculté de discernement lui fait nettement défaut et, se montrant incapable de rendre justice, il sombre dans la tyrannie. Cependant, il faut tenir compte du fait qu'à l'Acte IV Thésée se trouve sous le coup d'une passion déchaînée: la colère. Du reste, le statut passionnel de sa rage est lié métaphoriquement au «dragon impétueux» évoqué par Théramène dans son récit funèbre de la fin d'Hippolyte (v. 1519), car il est évident que le roi d'Athènes juge avec impétuosité. Par ailleurs, il faut noter les sentiments «(de) rage et de douleur» provoqués dans le monstre (v. 1531) (et dans Thésée) par Hippolyte qui se présente, dès lors, en «digne fils d'un héros» (v. 1527).

Déchiffrant mal le cœur de son fils, Thésée se livre à une condamnation systématique sans avoir pris conscience des preuves ni entendu les témoins. Il finit par assimiler Hippolyte aux «traîtres, (aux) ingrats sans honneur et sans loi» (v. 1147). S’identifiant par rapport à la Loi, il se sent profondément trahi. Thésée met en accusation alors la folie passionnelle d'un fils sacrilège. Enfermé dans son erreur, il impose à Hippolyte un exil irrémédiable et évoque la promesse de Neptune (vv. 1063-1068). Dans son aveu à son père, le fils s'avère en proie à l'asservissement des «lois» d'Aricie (v. 1123), d'où sa rébellion filiale. Dans ce dialogue de sourds, Hippolyte ne nie pas que sa flamme soit coupable et tient plutôt à affirmer l'exclusivité de son amour (vv. 1125-1126). Par suite de son jugement impétueux, Thésée passe de manière irréfléchie du projet d'exil de son fils à l'idée meurtrière d'une revanche divine. Il finit 
par maudire son fils en le soumettant aux lois d'une divinité vindicative. Mû par un courroux démesuré, le Roi adresse à Neptune une invocation irrévocable qui laisse pressentir la malédiction divine qui sera livrée par Phèdre à sa nourrice (IV, 6).

Quant à la calomnie d'CEnone, qui consiste à faire croire à Thésée que son fils a entrepris une tentative de viol envers Phèdre (IV, 1), il faut mettre en avant la complicité de la servante et de la reine. Prenant sur elle la faute tragique de Phèdre, Enone se livre à une dénonciation inspirée par des motifs méprisables mais Phèdre s'avère passivement consentante en lui aliénant sa volonté: «Fais ce que tu voudras, je m'abandonne à toi./Dans le trouble où je suis, je ne puis rien pour moi» (III, 3, vv. 911-912). Lors de sa «confession manquée» devant son mari, celle-ci ne parvient pas à innocenter Hippolyte $(\mathrm{IV}, 4)$. Alors que la reine était prête à s'accuser, Thésée lui a coupé la voix sans le savoir. Plus précisément, juste au moment où Phèdre allait lui avouer la vérité, Thésée fait part du fait qu'Aricie aime son fils: si la sœur des Pallantides dispose de la «foi» d'Hippolyte, c'est que celui-ci se soumet alors à la «loi» d'Aricie (v. 1187). Cette révélation funeste la frappe comme «un coup de foudre» (v. 1195) et fait éclater sa croyance mal fondée d'après laquelle Hippolyte était hostile envers les femmes ${ }^{19}$. Cette péripétie fait naître chez elle une prise de conscience épouvantable: «Hippolyte est sensible, et ne sent rien pour moi!» (v. 1203) et Aricie «... a fléchi son audace» (v. 1209) en vainquant son indifférence. Désignée, de nouveau, comme l'ennemie commune (cf., vv. 361-362), Aricie est érigée en criminelle, et Phèdre, jalouse, évoque sa race, qui a suscité le courroux de Thésée: elle cherche à tirer vengeance en exigeant, de la part du Roi, une peine exemplaire (vv. 1260-1261). La reine conçoit alors le vœu meurtrier de «perdre Aricie», et sa promptitude fait penser ironiquement à celle de Thésée. Dans cette rage destructrice, Phèdre subit toutefois un réveil de sa conscience morale car elle se révèle horrifiée par «(ses) homicides mains» et «l'imposture» de sa démarche hypocrite (vv. 1270-1271). Juge infernal, son père Minos va s'appliquer à soumettre impitoyablement sa fille dévergondée au terrible supplice cautionné par la loi divine ${ }^{20}$. N'ayant pu tirer aucune jouissance de son crime, la reine fait l'aveu pathétique d'avoir été incapable d'assouvir sa passion: «Jamais mon triste cœur n'a recueilli le fruit» (v. 1292).

Amorale, CEnone se montre prête à excuser l'«erreur» de sa maîtresse (v. 1296). Elle lui rappelle que l'amour entre Hippolyte et Aricie sera voué au malheur en raison de l'exil imposé par Thésée sur son fils (cf. «Ils ne se verront plus», v. 1252). Par ailleurs, Enone inscrit sa maîtresse dans l'humanité commune et finit par ramener son amour adultère à une trivialité: «L'amour n'a-t-il encor triomphé que de vous? | La faiblesse aux humains n'est que trop naturelle» (vv. 1300-1301). Dans son effort pour la consoler, la nourrice signale que même les dieux se sont livrés à des activités illicites (cf. «[les] feux illégitimes», v. 1306). Abattue et dans un état de choc comparable à celui de Thésée au début de l'Acte IV, Phèdre reproche à Enone ses mauvais conseils qui l'ont détournée du droit chemin. Dans sa malédiction, elle érige sa nourrice en coupable et la rabaisse à la lâcheté de son rang, une vilenie qui, à ses yeux, est inhérente à sa condition de servante (vv. 1319-1326).

En reprenant au dénouement son rôle de législateur royal, Thésée passe de l'éclaircissement (anagnorisis) (v. 1647) - cette scène de reconnaissance supposant la prise de conscience de son erreur tragique - à l'expiation de son crime (v. 1650).

(19) Soucieuse de justifier la froideur d'Hippolyte, Phèdre finit par le disculper en citant les «sauvages lois» auxquelles il obéissait dans la forêt (III, 1, v. 783).

(20) J. Scherer évoque justement «(le) délire de culpabilité où Phèdre s'imagine confrontée à des personnages terrifiants, comme ceux qu'on peut voir dans un cauchemar, et qui sont autant d'images du Père justicier: le Soleil, Jupiter, Minos» (1273-1288), Racine et/ou la cérémonie, Paris, Puf, 1982, p. 158. 
L'Acte $\mathrm{V}$ marque alors le retour de la Loi paternelle, qui valorise en même temps la restauration de l'Ordre patriarcal ${ }^{21}$. En adoptant Aricie, le Roi crée une alliance politique fondée sur «une nouvelle légalité»» ${ }^{22}$ et, à en croire M. Edwards, finit par «[guérir] la division de la Cité» ${ }^{23}$. Conformément à la tentative royale de Pyrrhus, qui entend faire table rase de son passé (Andromaque, IV, 5, v. 1344), Thésée fait vœu d'«oublier le passé».

Il convient maintenant de dégager les éléments constitutifs du dénouement tragique de Phèdre en fonction de la problématique de la Loi. Dans la mesure où Aricie finit par être l'héritière de Thésée, elle se trouve libérée. Vraie descendante de Pandion, elle dispose légalement des droits du sang, d'où la logique qui sous-tend son adoption par le Roi (v. 1654). Ce geste royal suppose, par ailleurs, la réconciliation des familles ennemies. Dans le cas de Phèdre, il va de soi qu'elle se rachète à la fin de la pièce, et cette confession ultime illustre le triomphe de la conscience morale sur la concupiscence $(\mathrm{V}, 7)$. L'aveu final de l'héroïne éponyme tient alors à son désir de se disculper de ses fautes - elle s'accuse d'inceste (v. 1624) - afin de préserver son honneur.

Après avoir fait preuve d'une quête d'éclaircissement auprès d'Aricie, Thésée, troublé, déchiffre mal la pâleur de la sœur des Pallantides (vv. 1414-1415). Mettant en valeur le jugement défectueux du Roi, celle-ci, grâce à une série de questions percutantes, montre à quel point il ignore le cœur de son fils: «Avez-vous de son cœur si peu de connaissance? | Discernez-vous si mal le crime et l'innocence?» (vv. 14291430): il s'avère le seul, selon elle, à ne pas voir la vertu exemplaire d'Hippolyte. Restant hostile à son égard, Thésée fonde ironiquement son jugement sur «des témoins certains» (v. 1441). S'étant mal renseigné jusqu'ici, dans son monologue (V, 4), il se montre soucieux d'«être mieux éclairci» (v. 1459). Son esprit profondément rongé, il se lamente sur la dimension équivoque de tout discours: «Quelle est donc sa pensée? et que cache un discours | Commencé tant de fois, interrompu toujours?» (vv. 14511452). En proie à un nouveau bouleversement - il s'agit du ravissement d'CEnone par la mer - ses impératifs à Panope se révèlent pathétiques, inutiles et tragiquement tardifs (vv. 1481-1483) - Thésée prend nettement conscience alors des limites cognitives de son jugement et souffre de sa propre crédulité (vv. 1485-1486). Il projette sur Théramène sa propre culpabilité et le gouverneur de son fils divulgue, lors de son récit funèbre $(\mathrm{V}, 6)$, qu'Hippolyte, sur le point de mourir, prévoit que son père sera, enfin, désabusé (v. 1563). À l'instar de Phèdre, l'appel aux dieux, chez Thésée, au nom du Désir et de la Loi, constitue une démarche funeste: «Ô mon fils! cher espoir que je me suis ravi! | Inexorables dieux, qui m'avez trop servi!» (vv. 1571-1572). Après avoir dénoncé la «victoire» de Phèdre, le Roi témoigne d'un manque de perception radical: il finit par brouiller la distinction entre le licite et l'illicite («Jouissez de sa perte, injuste ou légitime», v. 1598). Aussi signale-t-il un flou problématique entre ces concepts antinomiques ${ }^{24}$. On ne saurait trop insister sur la composante morale propre à ses multiples erreurs de jugement; de là, la prise de conscience de son injustice (v. 1609). Toutefois, c'est Phèdre qui va confesser en entraînant la rupture définitive du silence. S'attribuant à son tour l'iniquité de la mort d'Hippolyte, elle va finir par l'innocenter (vv. 1617-1619).

Nous avons voulu mettre en évidence la Loi en tant que conception doxale dans Phèdre, pièce qui recourt abondamment au lexique de la légalité et de la transgres-

(21) E. McMahon, Racine's "Phèdre", dans «Classics Incorporated», Birmingham, AL, Summa, 1998, p. 89.

(22) C. Spencer, La Tragédie du prince, Paris, Biblio 17, 1987 p. 294.

(23) M. Edwards, La Tragédie racinienne, Paris, La Pensée Universelle, 1972, p. 282.

(24) L. Horowitz, Racine's Laws cit., p. 141. 
sion. Afin de faire ressortir le décalage irrémédiable entre la Loi et le Désir, nous avons montré à maintes reprises que tout ce qui relève du Désir tend à subvertir la Loi, d'où l'impuissance radicale de la légalité à réprimer le désir. Tous les protagonistes éprouvent, à des degrés divers, un sentiment de culpabilité et tous visent à agir selon leur conscience. À cela s'ajoute leur effort pour soumettre autrui à l'impératif de leur désir, qui reste intrinsèquement criminel aux yeux de la Loi. Dans l'univers de Racine, force est d'admettre que l'amour est vécu comme une force de dégradation. Le poète tragique s'applique, du reste, à souligner la vulnérabilité des êtres à la pulsion érotique, ce qui donne lieu au spectacle funeste de l'amour non maitrisé. Face à l'impossibilité de dompter leurs pulsions libidinales, Phèdre et Hippolyte se montrent en proie au «fol amour» et se révèlent en même temps victimes de l'Ordre patriarcal en raison même de leur hérédité. Si l'on admet que la loi du Père s'oppose avec vigueur aux «amoureuses lois», il faut reconnaître que les impératifs de Vénus laissent entendre que Phèdre et Hippolyte doivent se plier inexorablement à leur destin. Pour en revenir à la sagesse de Théramène (I, 1), ceux qui se trouvent vaincus en amour n'ont d'autre option que de se soumettre aux lois de leur ennemie, Vénus: la condition humaine exige que l'on soit assujetti sous sa loi commune. La concupiscence relevant d'un comportement inhérent à toute l'humanité, on se rend compte de l'assujettissement collectif des mortels aux impératifs de la déesse de l'Amour.

Il convient de s'en remettre, au terme de cet essai, à la perspective freudienne de Francesco Orlando pour démontrer à quel point l'amour-passion dans Phèdre pousse les protagonistes à enfreindre les lois sociales fondamentales. Dans la mesure où la loi d'inceste apparaît comme la source de l'ensemble des règles sociales, le critique met en avant la valeur fondatrice du tabou de l'inceste, qui sert à assurer le bon fonctionnement de l'Ordre social: «La prohibition de l'inceste, ce premier désir, constitue la première loi humaine et, intériorisée, fonde toutes les autres règles, toutes les autres interdictions qui organisent la civilisation.... ${ }^{25}$. Étant donné la filiation profonde de l'héroïne tragique à l'égard de la Loi et du Désir, il est évident que l'inceste et l'adultère se ramènent aux interdits primordiaux de la Cité:

Loi et Désir, remords et regret ne sont plus seulement inséparables, ils se confondent l'un avec l'autre; et les reconnaître en leur identité confondue, comme voir le soleil sacré, devient pour Phèdre insoutenable si elle reste en vie (124). En définitive, la civilisation est fondée sur la répression patriarcale, notamment en ce qui concerne les lois fondatrices de la structure familiale. La prohibition de l'inceste s'inscrit, en dernière analyse, dans la Loi ${ }^{26}$.

RALPH ALBANESE

University of Memphis

(25) F. ORLANDO, Lecture freudienne de "Phèdre" cit., p. 35.

(26) Je tiens à remercier Ronald Tobin et Denis Grélé de leurs excellentes suggestions lors de l'élaboration de cet essai. 